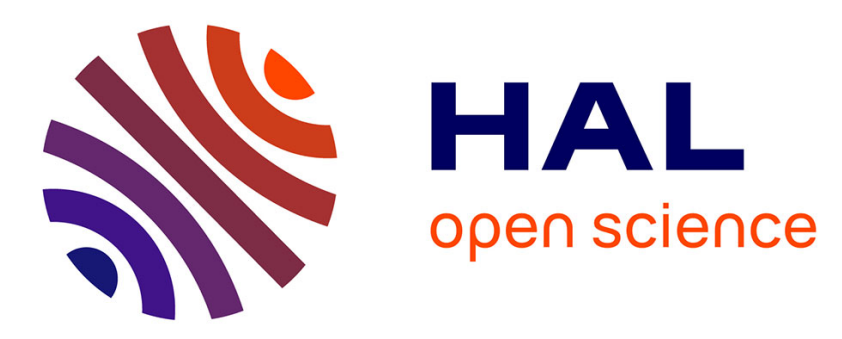

\title{
Non Linear Position and Closed Loop Stiffness Control for a Pneumatic Actuated Haptic Interface: the BirthSIM
}

Nicolas Herzig, Richard Moreau, Tanneguy Redarce, Frédéric Abry, Xavier Brun

\section{To cite this version:}

Nicolas Herzig, Richard Moreau, Tanneguy Redarce, Frédéric Abry, Xavier Brun. Non Linear Position and Closed Loop Stiffness Control for a Pneumatic Actuated Haptic Interface: the BirthSIM. 2015 IEEE IROS, Sep 2015, Hambourg, Germany. pp.1612-1618, 10.1109/IROS.2015.7353583 . hal01216564

\section{HAL Id: hal-01216564 \\ https://hal.science/hal-01216564}

Submitted on 23 Apr 2019

HAL is a multi-disciplinary open access archive for the deposit and dissemination of scientific research documents, whether they are published or not. The documents may come from teaching and research institutions in France or abroad, or from public or private research centers.
L'archive ouverte pluridisciplinaire HAL, est destinée au dépôt et à la diffusion de documents scientifiques de niveau recherche, publiés ou non, émanant des établissements d'enseignement et de recherche français ou étrangers, des laboratoires publics ou privés. 


\title{
Non Linear Position and Closed Loop Stiffness Control for a Pneumatic Actuated Haptic Interface: the BirthSIM
}

\author{
Nicolas Herzig, Richard Moreau, Tanneguy Redarce, Frédéric Abry and Xavier Brun
}

\begin{abstract}
This paper presents the BirthSIM model, a haptic interface of a childbirth simulator. The aim of this haptic interface is to improve haptic rendering and increase the biofidelity of the movements and forces which occur during the fetal descent. Thus the model of this 2 degrees of freedom pneumatic actuated interface is presented in this article, in order to synthesize a control law based on the backstepping method. The control law obtained allows targeted trajectories of positions and pneumatic stiffness to be tracked but also allows the equivalent stiffness of the end effector in Cartesian coordinates to be adapted in real time. The difference between pneumatic stiffness and closed loop stiffness is explained. Finally, some simulation results are presented to validate the control law behavior.
\end{abstract}

\section{INTRODUCTION}

Fortunately, most childbirths do not require practitioner intervention. But in some cases the obstetricians and the midwives have to be prepared to face different issues. On the other hand, C-sections are more and more used, to the detriment of other tools like forceps or vacuums. The lack of use of these tools leads to a loss of some skills and technical gestures, whereas they are more appropriate than C-sections in some cases. The BirthSIM and some other childbirth simulators have been developed in order to answer this issue and give to the practitioner a way to learn and practice some specific gestures used during delivery.

Different approaches have been discussed to design a childbirth simulator. Some simulators only use virtual reality [1] lack of a haptic interface decreases realism and does not allow forces during the gesture to be evaluated. Therefore, most childbirth simulators include a haptic interface. Different designs of haptic interfaces have been developed, for instance Sielhorst et al. [2] have decided to use a 6 degrees of freedom (DOF) industrial robot. The benefit of this kind of robot is that they are accurate and easy to use, but they are expensive and the effort ranges of the actuators are often over-sized to be used as haptic interface of a medical simulator. On the other hand, Abate et al. [3] have chosen an exoskeleton for haptic interface. This kind of haptic interface is a good way to reproduce hand and finger haptic sensations, but it is less suitable to simulate tool-assisted delivery.

Kheddar et al. have concluded in [4] that some industrial haptic interfaces appear to be limited and that developing a dedicated interface seems to be more suitable to simulate childbirth delivery. The BirthSIM has been developed in this

N. Herzig, R. Moreau, T. Redarce, F. Abry and X. Brun are with the Laboratoire Ampère, UMR CNRS 5005, Université de Lyon, INSA-Lyon, F-69621 Villeurbanne Cedex, France (e-mail: firstname.lastname@insalyon.fr). way. At first the BirthSIM has been designed especially to practice forceps-assisted delivery, but with the different improvements, the current version of this simulator is able to simulate different scenarios in which forceps are needed or not [5][6]. The latest version of the BirthSIM is 4 DOF robot actuated by two pneumatic cylinders and two motors. The pneumatic actuators have been chosen not only because the range of effort and displacement which are suitable to reproduce childbirth but also for their natural compliance which is suitable to simulate soft tissues behavior. The design, direct geometric model (DGM) and inverse geometric model (IGM) of the latest version of the BirthSIM have been presented in [7].

This paper will present the BirthSIM model which will be used to design a control law. In the first part, the global hardware architecture will be explained. The second part will describe the different steps to model the haptic interface and the hypothesis which have been chosen. The developed control law, which is presented in this paper, is a stiffness control based on the nonlinear theory which allows a targeted trajectory to be reached but also allows the stiffness of the actuator to be tuned in real time. These controlled pneumatic actuators can be used as variable stiffness actuators. It enables a better haptic feedback and best simulates the delivery behavior. The last parts will deal with the control law synthesis and discuss the future works.

\section{HARDWARE DESIGN OF THE BIRTHSIM}

As introduced in the previous section, the BithSIM is an haptic interface which simulate childbirth delivery. To be more precise, this robot is design to reproduce the fetal head movements during its descent inside the maternal pelvis. According to the obstetricians and the midwives, four DOF of the fetal head seem to be more important. This four DOF are the two translations of the center of the fetal head inside the sagittal plane of the maternal pelvis, the flexion of the fetal head and finally the internal rotation. That is the reason why the BirthSIM have been designed to reproduce those DOF but in this paper, the two last rotations will not be taken into account. Indeed, the stiffness control synthesis is more suitable for the pneumatic cylinder and does not concern the two rotations. The two neglected electric motors will actuate the end effector orientation and these rotations does not affect the dynamic behavior presented in section III. The position control of these two motors is based on a classical linear approach and have been widely addressed in literature. So, in order to simplify the study, the two motor and rotation have been removed. Fig. 1 shows the equivalent 
kinematic scheme of the BirthSIM, without the two motors. The two prismatic joints model the two pneumatic cylinders. The cylinder which consists of links 3 and 4 will be denoted cylinder 1 . Cylinder 2 consists of links 1 and 2 .

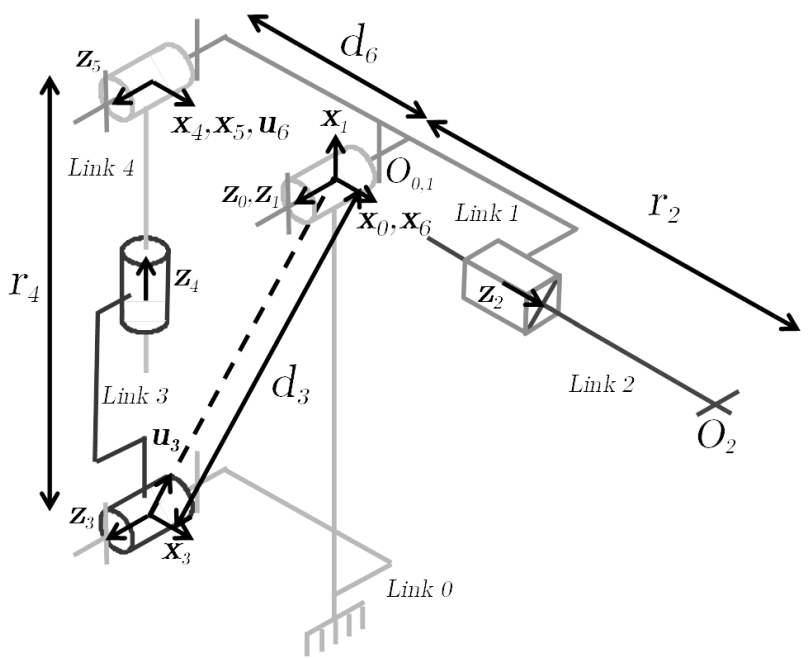

Fig. 1: Kinematic scheme of the BirthSIM's simplified architecture

Fig. 2 provides the global hardware architecture. The two actuators are two Festo pneumatic cylinders. The main characteristics are given in table I.

TABLE I: Main characteristics of the cylinder

\begin{tabular}{|c|c|c|}
\hline Reference & DSNU-25-400-PPV-A-Q & DSNU-25-200-PPV-A \\
\hline Notation & cylinder 2 & cylinder 1 \\
\hline Position & horizontal & vertical \\
\hline Stroke & $400 \mathrm{~mm}$ & $200 \mathrm{~mm}$ \\
\hline Piston diameter & $25 \mathrm{~mm}$ & $25 \mathrm{~mm}$ \\
\hline $\begin{array}{c}\text { Theoretical force at } \\
6 \text { bar, advancing }\end{array}$ & $295 \mathrm{~N}$ & $295 \mathrm{~N}$ \\
\hline $\begin{array}{c}\text { Theoretical force at } \\
6 \text { bar, retracting }\end{array}$ & $247 \mathrm{~N}$ & $247 \mathrm{~N}$ \\
\hline Rod geometry & square & cylinder \\
\hline
\end{tabular}

Each chamber of each cylinder is supplied by a independent Festo MPYE-5-M5-010-B proportional servovalve. A 5/3-way proportional servovalve ${ }^{1}$ to supply only one chamber is not necessary but in the final version only one servovalve per cylinder will be used so a 5/3-way proportional servovalve will then be necessary. Four pressure sensors are used to measure the pressure of each chamber. The end effector position and rotation are tracked by a 6 DOF Ascension trakSTAR magnetic tracker.

The control board chosen for the BirthSIM is a $A S P A C E$ board which allows sensors signals to be acquired and servovalves control to be generated. The code is generated with Matlab/Simulink which is suitable for control prototyping.

\footnotetext{
15/3-way proportional servovalve means that the valve has 5 inputs/outputs and 3 positions, proportional means that the spool position is proportional to the voltage applied to the valve
}

\section{MODEL}

In this section, the goal is to present the appropriate model of the BirthSIM to deduce a control law. The model is obtained by taking into account the mechanical behavior of the robot but also the thermodynamic behavior of the pneumatic part.

\section{A. Geometric model}

The DGM and IGM of the BirthSIM have been presented in [7]. In this paper, only the two prismatic active joints defined by the parameters $r_{4}$ and $r_{2}$ (cf. Fig. 1) are considered. To obtain the geometric model, the Khalil and Kleinfinger method have been used [8]. This method is known to be suitable for closed-loop robots modeling. In the procedure, each closed-loop is cut at a passive joint and a cut joint variable is introduced using a modified Denavit-Hartenberg parameterization. Finally, equating the two ending frames generates the geometric constraint equations. Table II gives the geometric parameters of the simplified architecture of the BirthSIM:

TABLE II: Geometric parameters of the BirthSIM

\begin{tabular}{|c|c|c|c|c|c|c|c|c|c|}
\hline$j$ & $a(j)$ & $\sigma$ & $\mu$ & $\mathrm{b}$ & $\mathrm{d}$ & $\mathrm{r}$ & $\gamma$ & $\alpha$ & $\theta$ \\
\hline \hline 1 & 0 & 0 & 0 & 0 & 0 & 0 & 0 & 0 & $\theta_{1}$ \\
\hline 2 & 1 & 1 & 1 & 0 & 0 & $r_{2}$ & 0 & $\frac{\pi}{2}$ & 0 \\
\hline 3 & 0 & 0 & 0 & 0 & $-d_{3}$ & 0 & $\gamma_{3}$ & 0 & $\theta_{3}$ \\
\hline 4 & 3 & 1 & 1 & 0 & 0 & $r_{4}$ & 0 & $-\frac{\pi}{2}$ & 0 \\
\hline 5 & 4 & 0 & 0 & 0 & 0 & 0 & 0 & $-\frac{\pi}{2}$ & $\theta_{5}$ \\
\hline 6 & 1 & 2 & 0 & 0 & $d_{6}$ & 0 & $-\frac{\pi}{2}$ & 0 & 0 \\
\hline
\end{tabular}

For the next section, the passive joint variable $\theta_{1}$ and the cut joint variable $\theta_{5}$ have to only be expressed with the active joint variables $r_{4}$ and $r_{2}$. To do so the geometric constraint equations have to be solved. These equations model the behavior of the closed loop made up of the links $0,1,3$, and 4. After solving these equations, the solutions obtained are

$$
\begin{aligned}
& \theta_{1}=\gamma_{3}-\arcsin \left(\frac{r_{4}^{2}-d_{3}^{2}-d_{6}^{2}}{2 d_{3} d_{6}}\right), \\
& \theta_{5}=\arcsin \left(\frac{r_{4}^{2}+d_{6}^{2}-d_{3}^{2}}{2 d_{3} r_{4}}\right) .
\end{aligned}
$$

To simplify the further equation, new variables are defined for the piston positions of cylinder 1 and 2 respectively, $y_{1} \in\left[-\frac{l_{1}}{2} ; \frac{l_{1}}{2}\right]$ and $y_{2} \in\left[-\frac{l_{2}}{2} ; \frac{l_{2}}{2}\right]$, with $l_{1}$ and $l_{2}$ the strokes of cylinders. $y_{1}$ and $y_{2}$ are respectively the piston position of cylinder 1 and 2 . So there exist $\left(k_{1}, k_{2}\right) \in \mathbb{R}^{2}$ such that

$$
r_{4}=y_{1}+k_{1}, \quad r_{2}=y_{2}+k_{2} \text {. }
$$

\section{B. Dynamic Model}

This section will deal with the dynamic behavior of the robot. The objective is to obtain a model suitable for control synthesis. In order to simplify the control design and reduce the time spent on parameters identification, some assumptions have been made. The assumptions are as follow

- All the links are supposed to be rigid bodies

- All the revolute joints are supposed to be perfect 


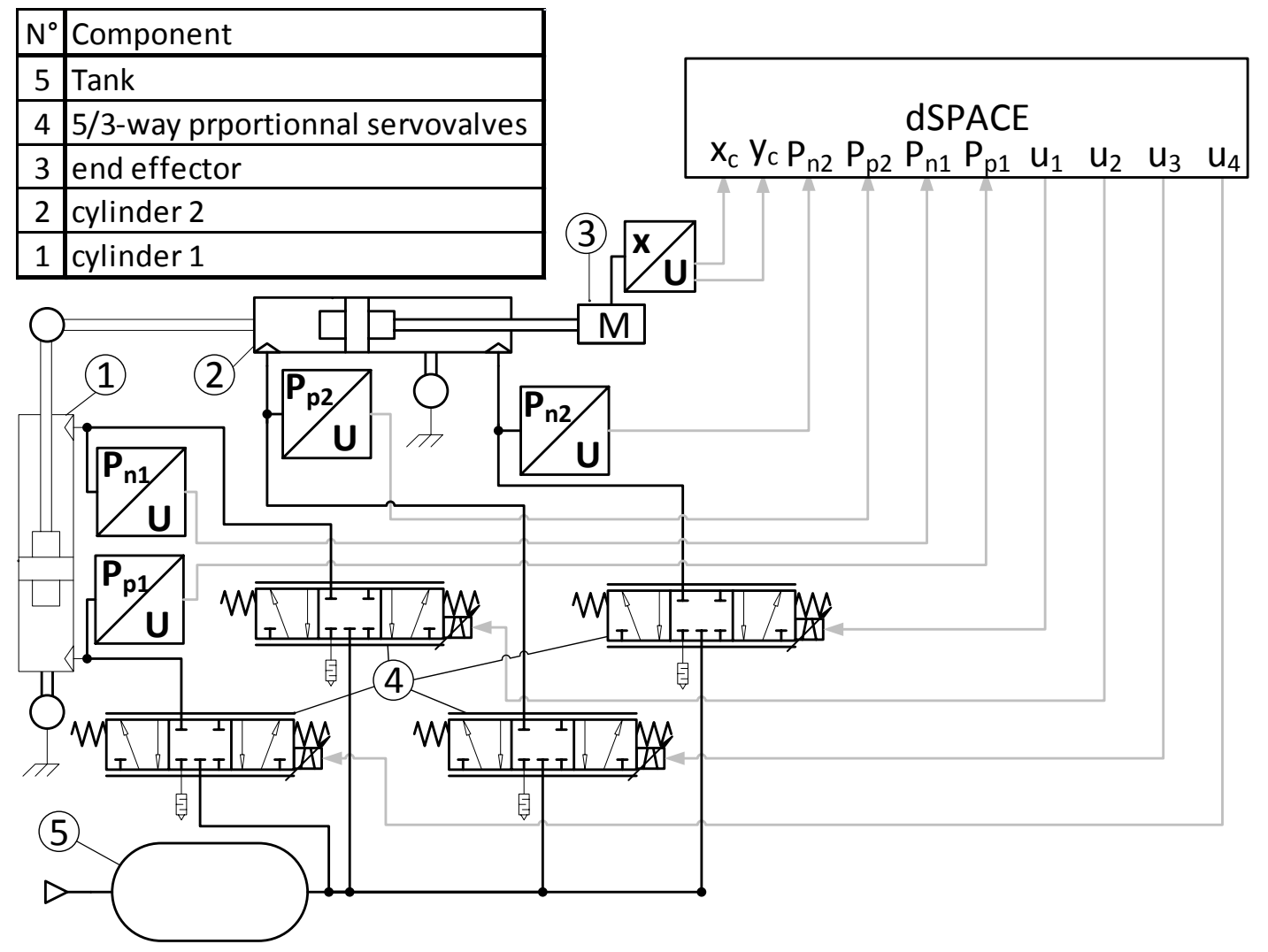

Fig. 2: Hardware architecture of the BirthSIM

- End effector is modeled as a point mass at $O_{2}(c f$. Fig. 1)

- All other masses and moments of inertia are neglected compared to that of the end effector

In this case, the forces taken into account are the weight of the end effector $W$, the pneumatic forces applied by the two cylinders $F_{\text {pneu } 1}$ and $F_{\text {pneu } 2}$, the forces applied by the external pressure $F_{\text {pext } 1}$ and $F_{\text {pext } 2}$, the friction forces $F_{f 1}$ and $F_{f 2}$ applied on cylinder 1 and 2 respectively.

The pneumatic forces of the cylinder can be deduced from the chambers' pressure measures whereas the external pressure forces only depend on the atmospheric pressure, which is assumed to be constant

$$
\begin{aligned}
& F_{\text {pneui }}=P_{p i} S_{p i}-P_{n i} S_{n i}, \\
& F_{\text {pext } i}=P_{a t m}\left(S_{p i}-S_{n i}\right) .
\end{aligned}
$$

$P_{p i}$ and $P_{n i}$ are respectively the pressures in $\mathrm{P}$ and $\mathrm{N}$ chamber of the $i$ cylinder, $P_{a t m}$ the atmospheric pressure, $S_{p i}$ and $S_{n i}$ are the effective piston areas in chamber P and $\mathrm{S}$ of the $i$ cylinder.

The friction model for the two prismatic joints is composed of Coulomb friction with viscous friction. Indeed, the friction force for cylinder $i$ is written

$$
F_{f i}=F_{c i} \operatorname{sign}\left(v_{i}\right)+F_{v i} v_{i},
$$

with $v_{i}=d y_{i} / d t$ the velocity of cylinder $i$ piston, $F_{c i}$ and $F_{v i}$ the Coulomb and viscous friction parameters of cylinder $i$. Then with the Newton-Euler method, the time derivative of $v_{1}$ and $v_{2}$ can be deduced

$$
\left\{\begin{array}{l}
\frac{d v_{2}}{d t}=\frac{F_{\text {pneu } 2}-F_{\text {pext } 2}-F_{f 2}}{m}+g \cos \left(\theta_{1}\right) \\
+\frac{4\left(y_{2}+k_{2}\right)\left(y_{1}+k_{1}\right)^{2} v_{1}^{2}}{4 d_{3}^{2} d_{6}^{2}-\left(\left(y_{1}+k_{1}\right)^{2}-d_{3}^{2}-d_{6}^{2}\right)^{2}} \\
\frac{d v_{1}}{d t}=\frac{\sqrt{4 d_{3}^{2} d_{6}^{2}-\left(\left(y_{1}+k_{1}\right)^{2}-d_{3}^{2}-d_{6}^{2}\right)^{2}}}{2\left(y_{2}+k_{2}\right)\left(y_{1}+k_{1}\right)} \\
\left(g \sin \left(\theta_{1}\right)-\frac{d_{6} \cos \left(\theta_{7}\right)}{m\left(y_{2}+k_{2}\right)}\left(F_{f 1}+F_{\text {pext } 1}-F_{\text {pneu } 1)}\right)\right. \\
-\frac{2\left(y_{1}+k_{1}\right)\left(\left(y_{1}+k_{1}\right)^{2}-d_{3}^{2}-d_{6}^{2}\right) v_{1}^{2}}{4 d_{3}^{2} d_{6}^{2}-\left(\left(y_{1}+k_{1}\right)^{2}-d_{3}^{2}-d_{6}^{2}\right)^{2}} \\
-\frac{2 v_{1} v_{2}}{y_{2}+k_{2}}-\frac{v_{1}^{2}}{y_{1}+k_{1}}
\end{array},\right.
$$

where $g$ is the Earth gravity coefficient.

\section{Thermodynamic model}

A common model used in literature to model the thermodynamic behavior inside the pneumatic cylinder chamber is based on polytropic law. The hypothesis that the temperature variation in the chambers are negligible [9] is assumed to design a non linear control law. In that case the model 
obtained is the following

$$
\left\{\begin{array}{l}
\frac{d P_{p i}}{d t}=\frac{k}{S_{p i} L_{p i}\left(y_{i}\right)}\left(r T q_{m p i}-P_{p i} S_{p i} v_{i}\right) \\
\frac{d P_{n i}}{d t}=\frac{k}{S_{n i} L_{n i}\left(y_{i}\right)}\left(r T q_{m n i}+P_{n i} S_{n i} v_{i}\right)
\end{array},\right.
$$

with

$$
L_{n i}\left(y_{i}\right)=\frac{l_{i}}{2}-y_{i}, \quad L_{p i}\left(y_{i}\right)=\frac{l_{i}}{2}+y_{i},
$$

where $k$ is the polytropic coefficient chosen experimentally, $l_{i}$ is the stroke of the cylinder $i, r$ is the specific gas constant for dry air, $T$ is the ambient temperature, $q_{m p i}$ and $q_{m n i}$ are the respective mass flow rates defined as positive while entering into the $i$ cylinder chambers $\mathrm{P}$ and $\mathrm{N}$.

\section{CONTROL}

\section{A. Control Model}

Currently the method considered for the control law is based on multi-input multi-output (MIMO) backstepping. This method is suitable to synthesize nonlinear control law with a real time closed loop stiffness tuning. Backstepping is based on a recursive method which designs a stabilizing function state by state using the Lyapunov theory. To apply this method, it is suitable to write the model in a strict feedback form [10][11]. The strict feedback form is described by the following equations

$$
\left\{\begin{array}{cc}
\dot{\xi}_{j}=f_{\xi_{j}}\left(\xi_{i}, \ldots, \xi_{n_{\xi}}\right)+g_{\xi_{j}}\left(\xi_{i}, \ldots, \xi_{n_{\xi}}\right) x_{1} & \forall j \in\left\{1, \ldots, n_{\xi}\right\} \\
\dot{x}_{i}=f_{i}\left(\xi_{i}, \ldots, \xi_{n_{\xi}}, x_{1}, \ldots, x_{i}\right) & \\
+g_{i}\left(\xi_{i}, \ldots, \xi_{n_{\xi}}, x_{1}, \ldots, x_{i}\right) x_{i+1} & \forall i \in\{1, \ldots, n\} \\
\dot{x}_{n+i}=f_{n+i}\left(\xi_{i}, \ldots, \xi_{n_{\xi}}, x_{1}, \ldots, x_{n+m}\right) \\
+\sum_{j=1}^{m-i+1} g_{n+i, j}\left(\xi_{i}, \ldots, \xi_{n_{\xi}}, x_{1}, \ldots, x_{n+m}\right) u_{j} \\
\forall i \in\{1, \ldots, m\} \\
y_{1}=h_{1}\left(x_{1}\right) \\
y_{i}=h_{i}\left(x_{1}, \ldots, x_{n+i}\right) \\
\forall i \in\{2, \ldots, m\}
\end{array}\right.
$$

Where $\xi$ and $x$ are the states of the system, $u$ and $y$ are respectively the control inputs and outputs. Finally the $f$ and $g$ are non linear functions.

To obtain the model in strict feedback form, a change of variables is needed. Abry et al. have proposed the A-T transform [12]. This transformation is similar to the Park transform in electric motor control but adapted for the pneumatic cylinder. The A-T transform consists in replacing the two mass flow rates $q_{m p i}$ and $q_{m n i}$ by two virtual flow rates $q_{m A i}$ and $q_{m T i}$ which respectively are the active and pressurization mass flow rates. This is the transformation for a single rod cylinder

$$
\left[\begin{array}{l}
q_{m A i} \\
q_{m T i}
\end{array}\right]=\frac{l_{i}}{2}\left[\begin{array}{cc}
\frac{1}{L_{p i}\left(y_{i}\right)} & -\frac{1}{L_{n i}\left(y_{i}\right)} \\
\frac{1}{L_{p i}\left(y_{i}\right)} & \frac{1}{L_{n i}\left(y_{i}\right)}
\end{array}\right]\left[\begin{array}{c}
q_{m p i} \\
q_{m n i}
\end{array}\right]
$$

By using this transformation it is possible to change the state model which describes the thermodynamic behavior, the new states are $F_{\text {pneui }}$ and $K_{\text {pneui }} . K_{\text {pneui }}$ is the pneumatic stiffness of the $i$ actuator. It could be noted that the same states have also been used in other works as in [13] where a force and pneumatic stiffness control synthesis is proposed.

$$
K_{\text {pneui }}=k\left(\frac{P_{p i} S_{p i}}{L_{p i}\left(y_{i}\right)}+\frac{P_{n i} S_{n i}}{L_{n i}\left(y_{i}\right)}\right),
$$

so the pneumatic model becomes

$$
\left\{\begin{array}{l}
\frac{d F_{\text {pneui }}}{d t}=\frac{2 k r T}{l_{i}} q_{m A i}-K_{\text {pneui }} v_{i} \\
\frac{d K_{\text {pneui }}}{d t}=\frac{A_{1} v_{i} y_{i} K_{\text {pneui }}-A_{2} v_{i} F_{\text {pneui }}-B_{1 i} y_{i} q_{m A i}}{L_{p i}\left(y_{i}\right) L_{n i}\left(y_{i}\right)} \\
+\frac{B_{2} q_{m T i}}{L_{\text {pi }}\left(y_{i}\right) L_{n i}\left(y_{i}\right)}
\end{array}\right.
$$

$$
\begin{array}{ll}
A_{1}=2(k+1), & A_{2}=k(k+1), \\
B_{1 i}=\frac{2 k^{2} r T}{l_{i}}, & B_{2}=k^{2} r T,
\end{array}
$$

with the different models obtained in the section III and the change of variables, the state model can now be built from the following state and control vector $\mathbf{X}$ and $\mathbf{U}$

$$
\mathbf{X}=\left[\begin{array}{llllllll}
x_{1} & x_{2} & x_{3} & x_{4} & x_{5} & x_{6} & x_{7} & x_{8}
\end{array}\right]^{T}, \quad \mathbf{U}=\left[\begin{array}{llll}
u_{1} & u_{2} & u_{3} & u_{4}
\end{array}\right]^{T},
$$

where

$$
\begin{array}{lll}
x_{1}=y_{2}, & x_{5}=y_{1}, & u_{1}=q_{m A 2}, \\
x_{2}=v_{2}, & x_{6}=v_{1}, & u_{2}=q_{m T 2}, \\
x_{3}=F_{\text {pneu } 2}, & x_{7}=F_{\text {pneu } 1}, & u_{3}=q_{m A 1}, \\
x_{4}=K_{\text {pneu } 2}, & x_{8}=K_{\text {pneu } 1}, & u_{4}=q_{m T 1} .
\end{array}
$$

Choosing this state allows to control not only the pneumatic force or position, but also the pneumatic stiffness of the two pneumatic actuators [14]. The state model can be deduced from (5) and (10)

$$
\left\{\begin{array}{l}
\dot{x}_{1}=x_{2} \\
\dot{x}_{2}=f_{2}\left(x_{1}, x_{2}\right)+g_{2} x_{3}+\tilde{f}_{2}\left(x_{1}, x_{5}, x_{6}\right) \\
\dot{x}_{3}=g_{3}\left(x_{2}\right) x_{4}+g_{3,1} u_{1} \\
\dot{x}_{4}=f_{4}\left(x_{1}, x_{2}, x_{3}, x_{4}\right)+g_{4,1}\left(x_{1}\right) u_{1}+g_{4,2}\left(x_{1}\right) u_{2} \\
\dot{x}_{5}=x_{6} \\
\dot{x}_{6}=f_{6}\left(x_{1}, x_{2}, x_{5}, x_{6}\right)+g_{6}\left(x_{1}, x_{5}\right) x_{7} \\
\dot{x}_{7}=g_{7}\left(x_{6}\right) x_{8}+g_{7,3} u_{3} \\
\dot{x}_{8}=f_{8}\left(x_{5}, x_{6}, x_{7}, x_{8}\right)+g_{8,3}\left(x_{5}\right) u_{3}+g_{8,4}\left(x_{5}\right) u_{4}
\end{array}\right.
$$

The model defined by (13) does not respect the strict feedback form presented in (7) because of the $\tilde{f}_{2}$ part.

$$
\tilde{f}_{2}=\underbrace{\frac{4\left(y_{2}+k_{2}\right)\left(y_{1}+k_{1}\right)^{2} v_{1}^{2}}{4 d_{3}^{2} d_{6}^{2}-\left(\left(y_{1}+k_{1}\right)^{2}-d_{3}^{2}-d_{6}^{2}\right)^{2}}}_{\text {centrifugal acceleration }}+\underbrace{g \cos \left(\theta_{1}\right)}_{\text {weight projection }} .
$$

In the case of the BirthSIM robot, $\tilde{f}_{2}$ is bounded. Indeed, because of the low dynamic of the delivery, the speed of the 
first cylinder stays low. That is the reason why the centrifugal acceleration is considered to be bounded. On the other hand, the weight projection on $\mathbf{z}_{2}$ (cf. Fig. 1) is also bounded in the robot workspace. In the subsection III-A, it has been shown that $\theta_{1}$ is bounded around $\pi / 2$ so the weight projection is bounded around zero. So in the next section the model used to synthesize the control is the one given in (13) but without $\tilde{f}_{2}$.

\section{B. Controller synthesis}

As it has been explained, the backstepping method has been used to obtain the control law. Using two servovalves for each cylinder allows two independent trajectories for each cylinder to be tracked, that is four for BirthSIM robot. Two DOF will be used to track the desired position of the pistons $y_{d 1}$ and $y_{d 2}$ respectively for cylinder 1 and 2 . To define the trajectory the derivatives of these positions are needed, the desired velocities $v_{d 1}, v_{d 2}$, the desired accelerations $a_{d 1}, a_{d 2}$ and the desired jerks $j_{d 1}$ and $j_{d 2}$. The two left DOF will be used to track the desired pneumatic stiffnesses' trajectories, $K_{\text {pneud } 1}$ and $K_{\text {pneud } 2 \text {, defined by }}$ their derivative $d K_{\text {pneud1 }} / d t$ and $d K_{\text {pneud } 2} / d t$.

The method used to obtain the control law is the same as that presented by Abry et al. in [12][15] for the four first states and then reiterated for the following four. This method introduces some gains which will allow the closed loop stiffness of the actuators to be tuned. In electro pneumatic field, two different stiffnesses can be defined. The first one is the pneumatic stiffness defined in (9). This stiffness is a state of the system, it shows the actuator tendency counteract an external force. But the displacement due to this external force will depend of the piston position. The second one is the closed loop stiffness, this stiffness is a parameter which depends on the control law gains. By tuning this parameter, the behavior of the pneumatic actuator against an external disturbance (force) can be chosen. Indeed for a force $F_{\text {disturb }}$ applied on the actuator, the displacement of the piston will be

$$
\Delta y=\frac{F_{\text {disturb }}}{K_{c l}} .
$$

The Abry et al. method enable the control of the closed loop stiffness of each actuator, but for a robotic haptic interface, it is more useful to control the end effector equivalent stiffness. So it is necessary to compute the closed loop stiffness of both cylinder $K_{c l 1}$ and $K_{c l 2}$ from a desired end effector stiffness in cartesian space $K_{c l x}$ and $K_{c l y}$. To do that, the kinematic model is needed. The kinematic model is obtained by derivation of the geometric model

$$
\left[\begin{array}{l}
d x_{c} \\
d y_{c}
\end{array}\right]=J\left(y_{1}, y_{2}\right)\left[\begin{array}{l}
d y_{1} \\
d y_{2}
\end{array}\right],
$$

where $d x_{c}$ and $d y_{c}$ are respectively the cartesian end-effector coordinate $x_{c}$ and $y_{c}$ differentials and $J$ is the Jacobian matix of the robot.

Due to the force-velocity duality, with quasi-static assumption, the relation between external forces applied on endeffector $F_{u s x}$ and $F_{u s y}$ and equivalent forces on actuators
$F_{u s 1}$ and $F_{u s 2}$ is given by

$$
\left[\begin{array}{l}
F_{u s 1} \\
F_{u s 2}
\end{array}\right]=J^{T}\left[\begin{array}{l}
F_{u s x} \\
F_{u s y}
\end{array}\right] .
$$

It can be deduced that the equivalent closed loop stiffnesses are obtained by computing the next equations

$$
\begin{aligned}
& K_{c l 1}=\left[\begin{array}{l}
1 \\
0
\end{array}\right]^{T} J^{T}\left[\begin{array}{cc}
K_{c l x} & 0 \\
0 & K_{c l y}
\end{array}\right] J\left[\begin{array}{l}
1 \\
0
\end{array}\right], \\
& K_{c l 2}=\left[\begin{array}{l}
0 \\
1
\end{array}\right]^{T} J^{T}\left[\begin{array}{cc}
K_{c l x} & 0 \\
0 & K_{c l y}
\end{array}\right] J\left[\begin{array}{l}
0 \\
1
\end{array}\right] .
\end{aligned}
$$

The global architecture of the controller is shown by Fig. 3. The servovalve static characterizations are obtained experimentally. These characterizations give for each servovalve the voltage which has to be applied to obtain a desired mass flow for given pressure inside the chamber.

\section{SIMULATION}

In this section, the controller obtained by backstepping is tested in simulation. The objective is to validate the assumption taken in IV-A and to show that it is possible to follow a desired trajectory and to control the closed loop stiffness.

It is noteworthy that the model used for the simulation is more complex than the model used to synthesize the control law. Indeed, if the model presented in this paper are suitable and enough simple to synthesize the control law, they are not necessary the most accurate to model the system. More over in order to verify that the assumptions taken to simplify the control model does not impact the desired behavior of the system too much, using a more complex model for the simulation is suitable. Thus, the simulation model takes account of the centrifugal acceleration and the weight projection presented in (14). The dynamic model also includes the forces applied by the user on the haptic interface $F_{u s x}$ and $F_{u s y}$. The thermodynamic model used in the simulation is not a polytropic model with a constant temperature, but a model with heat exchange between the two chambers and between the chambers and the external environment. Finally, the servovalves are modeled as first order systems with an estimated time constant.

Fig. 4 shows simulation results. In this simulation the targeted trajectory is a circle in Cartesian coordinates. The user disturbance in cartesian coordinate and the setpoints of closed loop stiffness are shown on Fig. 4a. The same sequence is repeated twice, once with a closed loop stiffness set at $2000 \mathrm{~N} / \mathrm{m}$ on both cartesian axes $x_{c}$ and $y_{c}$ and the second time with a stiffness doubled. It is not obvious to define a targeted trajectory of pneumatic stiffness. For instance, this state could be used to optimize air consumption, but for the BirthSIM, this kind of objective is not a priority. In this paper, the main idea is to illustrate the difference between the closed loop stiffness and the pneumatic stiffness. For this reason, it has been chosen to reduce the pneumatic stiffness whereas the closed loop stiffness is increased in order to show that they are independent. Fig. $4 \mathrm{~b}$ presents the targeted and real trajectories of pneumatic stiffness for each cylinder. 


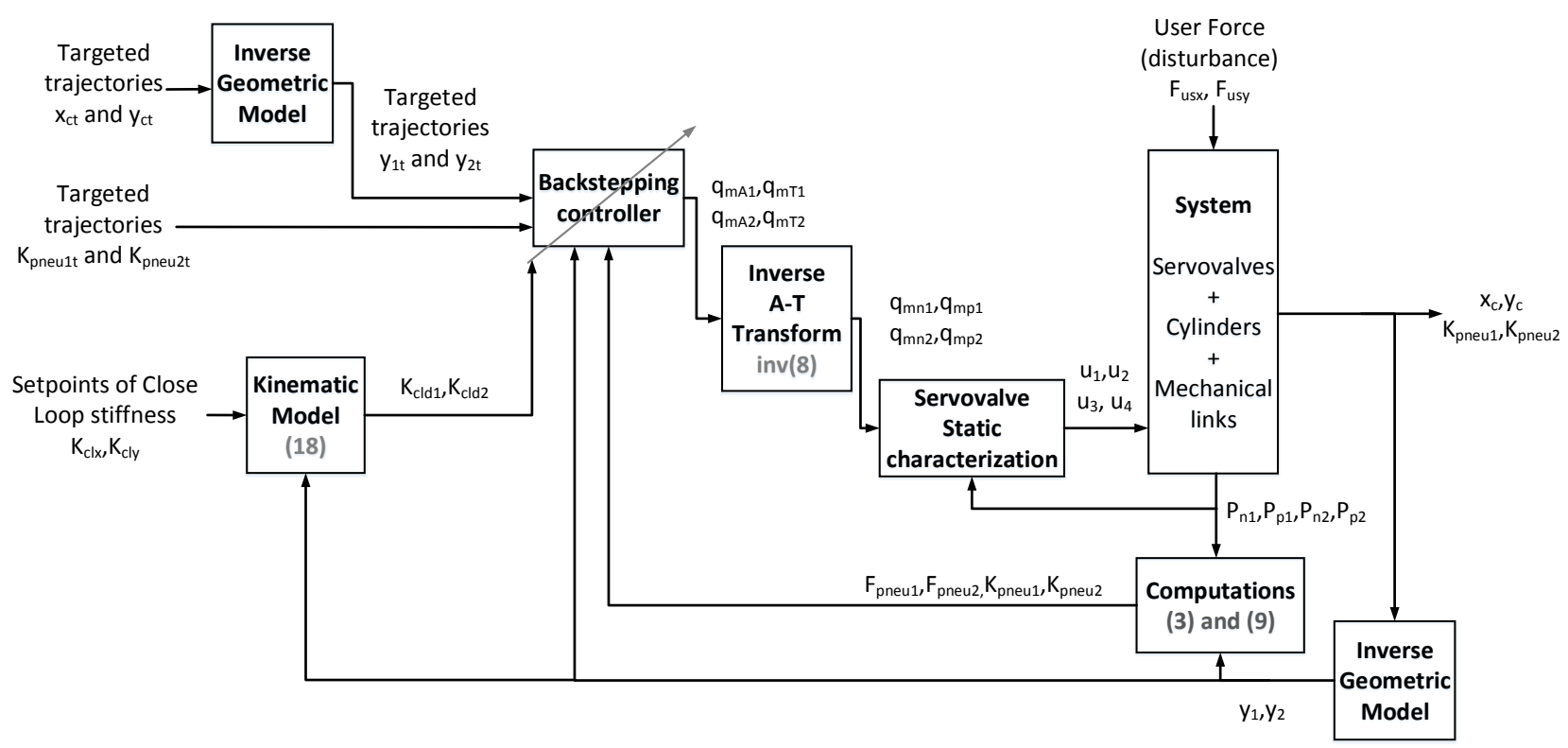

Fig. 3: Architecture of the controller

Fig. $4 \mathrm{c}$ shows the targeted trajectory and the real trajectory in cartesian space. And finally, Fig. $4 \mathrm{~d}$ shows the targeted trajectory and the real trajectory of the cartesian coordinates. In this figure the $x_{c k}$ and $y_{c k}$ are obtained by adding the estimated displacement due to disturbance to the targeted trajectories.

$$
\begin{aligned}
& x_{c k}=x_{c t}+\frac{F_{u s x}}{K_{c l x}}, \\
& y_{c k}=y_{c t}+\frac{F_{u s y}}{K_{c l y}} .
\end{aligned}
$$

In Fig. $4 c$ and $4 d$, the expected behavior is obtained. This simulation shows that it is possible to reduce the pneumatic stiffness of each actuator and also increase the closed loop stiffness. That is why it is important to distinguish them. Others scenarios have been played on this simulation. It has been noticed that, for lower disturbances, assuming that the weight effect on cylinder 2 can be neglected, too much error is induced. That is why, another control law is about to be developed with a weight compensation for cylinder 2 .

\section{CONCLUSIONS AND FUTURE WORKS}

The control model of a two DOF pneumatic robot has been presented. The difference between the closed loop stiffness and the pneumatic stiffness has been illustrated by a simulation. Finally, it has been shown that for this kind of robot it is possible to develop a control law which not only allows targeted trajectories of position and pneumatic stiffness to be tracked but also enable real time tuning of the closed loop stiffness.

In the near future, this control law will be tested experimentally on the BirthSIM robot. Some improvements will be added to the current control law. The first point will be to add a weight compensation for cylinder 2 . The second point is to reduce the number of servovalves. Indeed, the servovalves are expensive so it could be a way to reduce the price of a pneumatic robot. By removing a servovalve per cylinder, the control of pneumatic stiffness is lost but, the closed loop stiffness tuning can be preserved.

In order to use the BirthSIM as an haptic interface which reproduce childbirth delivery, some data of fetal head movement are needed to define the targeted trajectories of positions and the setpoints of closed-loop stiffness. Obtaining this data by in vivo measurement is difficult because of ethical issues. So to overcome this issue some partners of the SAGA project (French acronym for simulator to learn delivery gestures) work on a biomechanical model of childbirth delivery which will compute fetal head trajectories and will also allow the stiffness to be estimated [16][17][18]. Of course, all this data will be validated by expert practitioners.

\section{ACKNOWLEDGMENT}

The authors would like to thank the ANR French National Research Agency for financing the SAGA project (ANR-12MONU-0006).

\section{REFERENCES}

[1] R. Lapeer, M. Chen, and J. Villagrana, "Simulating obstetric forceps delivery in an augmented environment," in Augmented environments for Medical Imaging including Augmented Reality in Computer-aided Surgery, 2004.

[2] T. Sielhorst, T. Blum, and N. Navab, "Synchronizing 3d movements for quantitative comparison and simultaneous visualization of actions," in International Symposium on Mixed and Augmented Reality. IEEE, 2005, pp. 38-47.

[3] A. Abate, G. Acampora, V. Loia, S. Ricciardi, and A. Vasilakos, "A pervasive visual-haptic framework for virtual delivery training," Information Technology in Biomedicine, IEEE Transactions on, vol. 14, no. 2, pp. 326-334, March 2010.

[4] A. Kheddar, C. Devine, M. Brunel, C. Duriez, and O. Sibony, "Preliminary design of a childbirth simulator haptic feedback," in Intelligent Robots and Systems, vol. 4. IEEE, 2004, pp. 3270-3275 vol.4. 


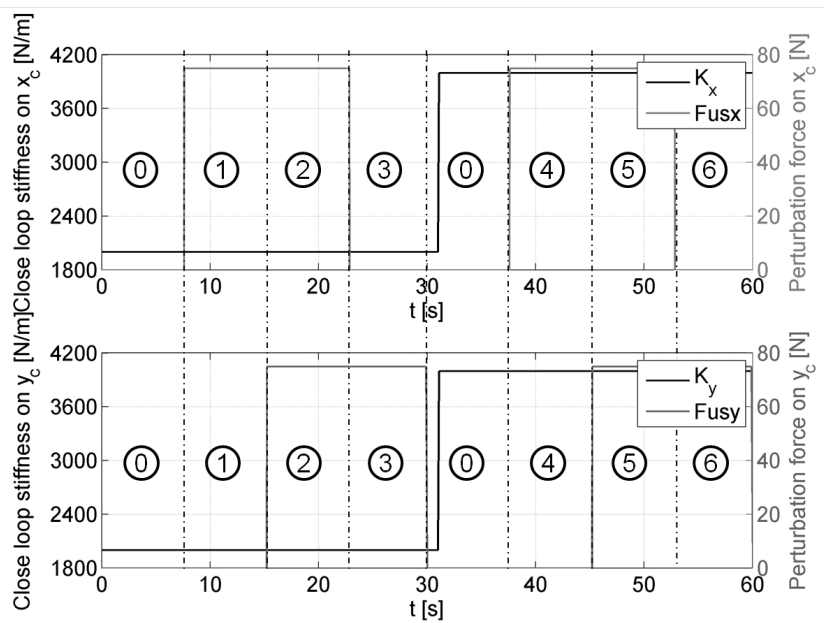

(a) User disturbance and closed loop stiffness setpoints

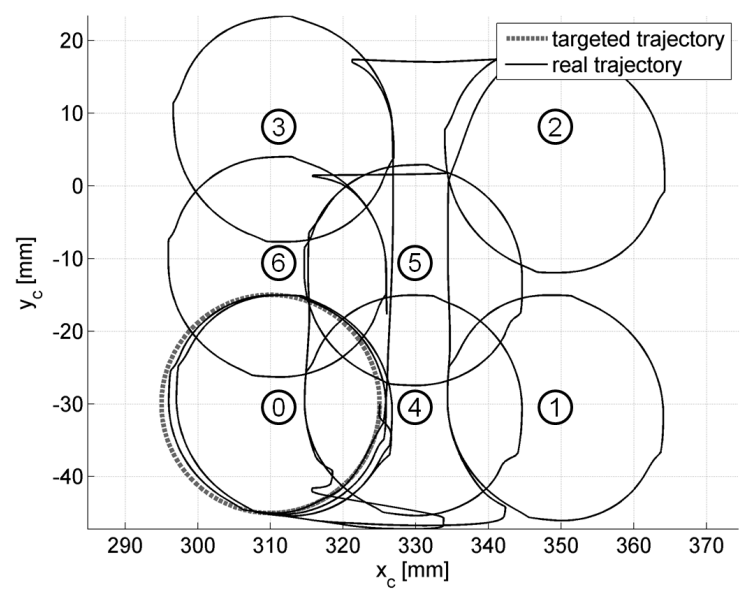

(c) Cartesian position
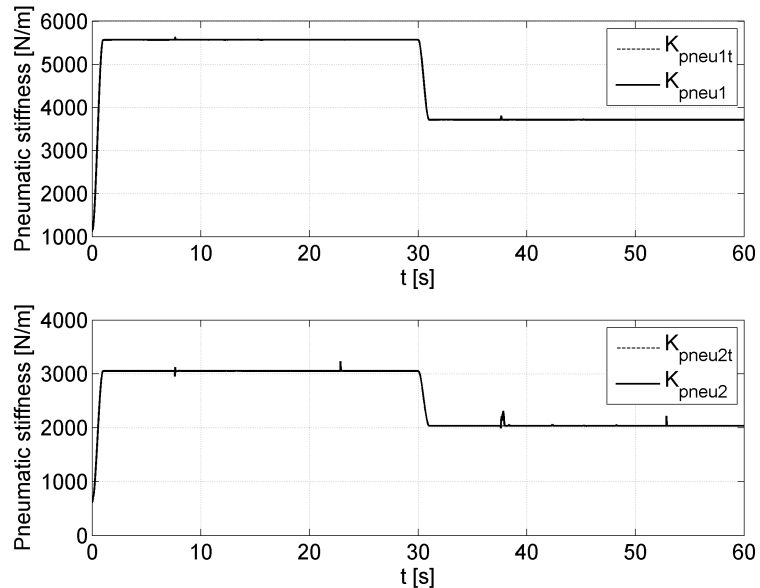

(b) Pneumatic stiffness
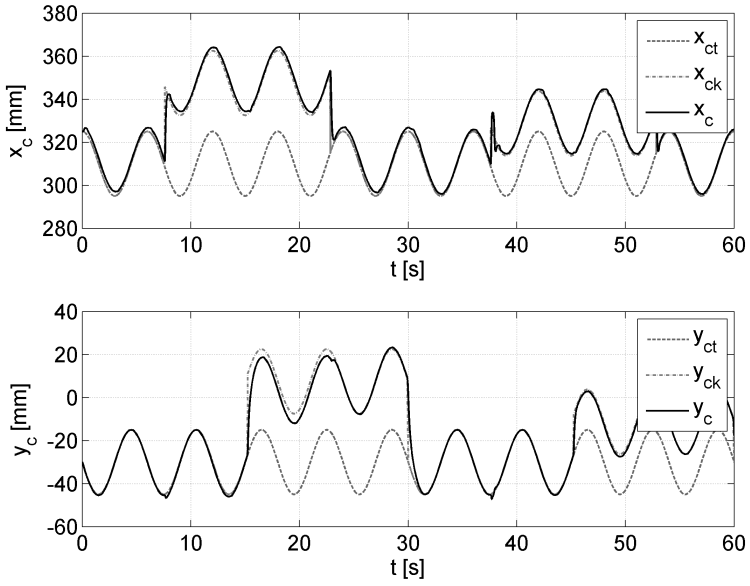

(d) Cartesian coordinates

Fig. 4: Simulation results

[5] R. Silveira, M. T. Pham, T. Redarce, M. Betemps, and O. Dupuis, "A new mechanical birth simulator: Birthsim," in Intelligent Robots and Systems, 2004. (IROS 2004). Proceedings. 2004 IEEE/RSJ International Conference on, vol. 4, 2004, pp. 3948-3953.

[6] R. Moreau, M. T. Pham, R. Silveira, T. Redarce, X. Brun, and O. Dupuis, "Design of a new instrumented forceps: Application to safe obstetrical forceps blade placement," Biomedical Engineering, IEEE Transactions on, vol. 54, no. 7, pp. 1280-1290, July 2007.

[7] N. Herzig, R. Moreau, and T. Redarce, "A new design for the birthsim simulator to improve realism," in Engineering in Medicine and Biology Society, 2014. EMBC 2014. Annual International Conference of the IEEE, 2014, pp. 2065-2068.

[8] W. Khalil and J. Kleinfinger, "A new geometric notation for open and closed-loop robots," in Robotics and Automation. Proceedings. 1986 IEEE International Conference on, vol. 3, no. 1174-1179. IEEE, 1986.

[9] B. Andersen, The analysis and design of pneumatic systems. Wiley, 1967.

[10] M. Krstic, P. V. Kokotovic, and I. Kanellakopoulos, Nonlinear and Adaptive Control Design, 1st ed. John Wiley \& Sons, Inc., 1995.

[11] H. K. Khalil, Nonlinear systems, 3rd ed. Prentice Hall, 2002.

[12] F. Abry, X. Brun, S. Sesmat, and E. Bideaux, "Non-linear position control of a pneumatic actuator with closed-loop stiffness and damping tuning," in Control Conference (ECC), 2013 European, 2013, Manuscript, pp. 1089-1094.
[13] B. Taheri, D. Case, and E. Richer, "Force and stiffness backsteppingsliding mode controller for pneumatic cylinders," Mechatronics, IEEE/ASME Transactions on, vol. 19, no. 6, pp. 1799-1809, Dec 2014.

[14] X. Shen and M. Goldfarb, "Simultaneous force and stiffness control of a pneumatic actuator," Journal of Dynamic Systems Measurement and Control-Transactions, vol. 129, no. 4, pp. 425-434, 2007.

[15] F. Abry, X. Brun, S. Sesmat, E. Bideaux, and C. Ducat, "Electropneumatic cylinder backstepping position controller design with real time closed-loop stiffness and damping tuning," Control Systems Technology, IEEE Transactions on, [accepted].

[16] R. Buttin, F. Zara, B. Shariat, T. Redarce, and G. Grang, "Biomechanical simulation of the fetal descent without imposed theoretical trajectory," Computer Methods and Programs in Biomedicine, vol. 111, no. 2, pp. $389-401,2013$.

[17] M. Bailet, F. Zara, and E. Promayon, "Shell finite element model for interactive foetal head deformation during childbirth," Computer Methods in Biomechanics and Biomedical Engineering, vol. 16, no. S1, pp 312-314, 2013.

[18] F. Goulette and Z.-W. Chen, "Fast computation of soft tissue deformations in real-time simulation with hyper-elastic mass links," Computer Methods in Applied Mechanics and Engineering, vol. 295, pp. 18 $38,2015$. 